\title{
TO STUDY THE ASSESSMENT OF LIPID ABNORMALITIES IN PATIENTS SUFFERING FROM GALLSTONES
}

\author{
Mahesh Gupta1, Vivek Singh², Pooja Gupta3, Preety Gupta4 \\ 1 Professor, Department of Surgery, Rama Medical College Hospital and Research Centre, Mandhana, Kanpur, U. P. \\ ${ }^{2}$ Associate Professor, Department of Anaesthesia, GCRG Institute of Medical Sciences, Lucknow, U. P. \\ 3 Senior Consultant, Department of Medicine, SGL Charitable Hospital, Jalandhar, Punjab. \\ ${ }_{4}^{4}$ M.Sc, Department of Biochemistry, Rama Medical College Hospital and Research Centre, Mandhana, Kanpur, U. P.
}

ABSTRACT
80 percent of gallstones are made of cholesterol, whereas 20 percent of gallstones are made of calcium salts and bilirubin.
Gallstone diseases being common disorders might have an association with abnormal lipids.
Aims and Objectives- This study is done to assess the lipid abnormalities in patients suffering from gallstone diseases.

Aims and Objectives- This study is done to assess the lipid abnormalities in patients suffering from gallstone diseases.

\section{MATERIALS AND METHODS}

The is a case control study, the sample size was taken for convenience during the study, and was carried out in the Department of Biochemistry among the healthy individuals and patients of gallstones attending the OPD of General Surgery, Rama Medical College Hospital and Research Centre, Mandhana, Kanpur (UP). The gallstones were collected after cholecystectomy. $5 \mathrm{~mL}$ of blood sample was collected after overnight fasting from each of the participants to measure the serum lipid parameters. Student's independent ttest was used to examine the degree of significance. $P$ values less than 0.05 was considered significant.

Limitations- Since the calculated sample size was too high and thereby not feasible to include in this limited period of study, we had to limit the sample size for convenience.

\section{RESULTS}

Levels of serum triglyceride and levels of VLDL are statistically significant $(\mathrm{p}<0.05)$, while the levels of serum cholesterol, HDL and LDL are not statistically significant $(\mathrm{p}>0.05)$. The results are depicted in different tables to compare the groups individually.

\section{CONCLUSION}

Although, this small sample size study concluded that there is correlation between presence of gallstones and TGs as well as VLDL, but a larger sample size study is required to further strengthen these risk factors.

\section{KEY WORDS}

Gallstones, Lipid Profile, Cholesterol (C), Low-Density Lipoprotein (LDL), High-Density Lipoprotein (HDL), Triglycerides, Very LowDensity Lipoprotein (VLDL).

HOW TO CITE THIS ARTICLE: Gupta M, Singh V, Gupta P, et al. To study the assessment of lipid abnormalities in patients suffering from gallstones. J. Evolution Med. Dent. Sci. 2018;7(21):2572-2575, DOI: 10.14260/jemds/2018/579

\section{BACKGROUND}

80 percent of gallstones are made of cholesterol, whereas 20 percent of gallstones are made of calcium salts and bilirubin ${ }^{1}$ and are known as pigment stones. Cholesterol stones may develop when there is too much cholesterol in the bile secreted by liver. Bile usually dissolves or breaks down cholesterol $^{2}$ and bilirubin is a chemical produced when liver destroys old red blood cells. Stones form when gallbladder cannot break down the excess bilirubin. Gallstone diseases being common disorder might have an association with abnormal lipids. Blood lipid profile is a panel of blood tests that serves as an initial broad medical screening tool for abnormalities in lipids such as cholesterol and triglycerides. ${ }^{3}$

'Financial or Other Competing Interest': None.

Submission 14-02-2018, Peer Review 05-05-2018,

Acceptance 12-05-2018, Published 21-05-2018.

Corresponding Author:

Dr. Vivek Singh,

Associate Professor

Department of Anaesthesia,

GCRG Institute of Medical Sciences,

Lucknow,

Uttar Pradesh.

E-mail: gm982003@gmail.com

DOI: $10.14260 /$ jemds $/ 2018 / 579$

(c) (1) $\odot$
The lipid profile typically includes Low-density lipoprotein (LDL), High-density lipoprotein (HDL), Triglycerides and Total cholesterol.4-7 Using their values, we may also calculate Very low-density lipoprotein (VLDL) and Cholesterol: HDL ratio. Our study is aimed in finding the lipid abnormalities in patients suffering from gallstone diseases.

\section{MATERIALS AND METHODS}

The case control study was used in the study and the sample size was taken for convenience during the study, which was carried out in the Department of Biochemistry among the healthy individuals and patients of gallstones attending to the OPD of General Surgery, Rama Medical College Hospital and Research Centre, Mandhana, Kanpur (UP). This study was conducted from 1st April 2016 to 31st December 2016.

Gallstones are solid particles that develop in the gallbladder. Stones are formed from the crystallisation of bile, a fluid made by the liver and secreted into the bowel through the bile ducts to help digest fats. The gallstones were collected after cholecystectomy.

Total subjects taken were 50 cases and 50 controls. These cases and controls were selected by using a standard questionnaire. It was used as a study tool to collect the data including basic profile of participants and the proforma also included the different types of investigations related to the 
study, i.e. lipid profile assessment, ultrasound of the abdomen. A verbal or a written consent was obtained from participants before the sample collection.

These cases and controls were selected from the patients who visited the Department of Biochemistry, Rama Medical College Hospital and Research Centre, Mandhana, Kanpur (UP).

All the patients were in the age group of 20 to 70 years with the mean age in cases was $43.2 \pm 13.69$ and in controls was $46.2 \pm 14.39$. Comparison of serum lipid profile between patients and control groups showed that the levels of serum total cholesterol $(147.22 \pm 38.71)$ in patients were slightly higher than that of the control group $(138.32 \pm 49.54)$, but there was no significant variation in total cholesterol ( $p>0.05$ ) between patient and control groups.

A standard questionnaire was used as study tool to collect the data including basic profile of participants and the proforma also included the different types of investigations related to the study, i.e. lipid profile assessment, ultrasound of the abdomen. A verbal or a written consent was obtained from participants before the sample collection. $5 \mathrm{~mL}$ of blood sample was collected after overnight fasting from each of the participants to measure the serum lipid parameters. Serum cholesterol levels were measured with Cholesterol Reagent (CHOD-PAP). Serum triglycerides were measured with Triglycerides DES Reagent (Dynamic Extended Stability with Lipid Clearing Agent GPO- Trinder Method, End Point). HDL Cholesterol were measured with ERBA Cholesterol Reagent. (Phosphotungstic Acid Method, End Point). LDL Cholesterol was measured by Friedewald equation and the betaquantification method. VLDL Cholesterol= TGs/5. The gallstones were collected after cholecystectomy. Statistical Software namely SPSS 21.0 version was used for the analysis of the data and Microsoft Word and Excel had been used to generate the graphs and tables. Descriptive statistics like mean, standard deviation and student's independent t-test was used to examine the degree of significance. $P$ values less than 0.05 was considered significant.

\section{RESULTS}

Total patients taken were 50 cases and 50 controls, patients participated in this study Cases i.e. patients with gallstone disease and Controls i.e. patients without gallstone disease. Out of these $26(52 \%)$ males and 24 (48\%) females were in case group and had gallstone disease whereas $33(66 \%)$ were males and 17 (34\%) were females in the control group. All the patients were in the age group of 20 to 70 years with mean age in cases was $43.2 \pm 13.69$ and in controls was 46.2 \pm 14.39 . Comparison of serum lipid profile between patients and controls group showed that the levels of serum total cholesterol $(147.22 \pm 38.71)$ in patients were slightly higher than that of the control group $(138.32 \pm 49.54)$, but there was no significant variation in total cholesterol $(p>0.05)$ between patients and controls group. LDL-C was even lower in patients $(74.74 \pm 28.84)$ as compared to control group (76.40 \pm 37.00$)$. HDL-C was slightly higher in patients $(47.78 \pm 15.47)$ than the control group $(44.06 \pm 15.65)$, but it was also not significant. In our study triglycerides $(126.34 \pm 64.83)$ and very low-density lipoprotein

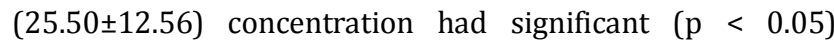
increase in patients with gallstone formation compared with control (105.66 \pm 38.55 and $21.26 \pm 7.96$ respectively).

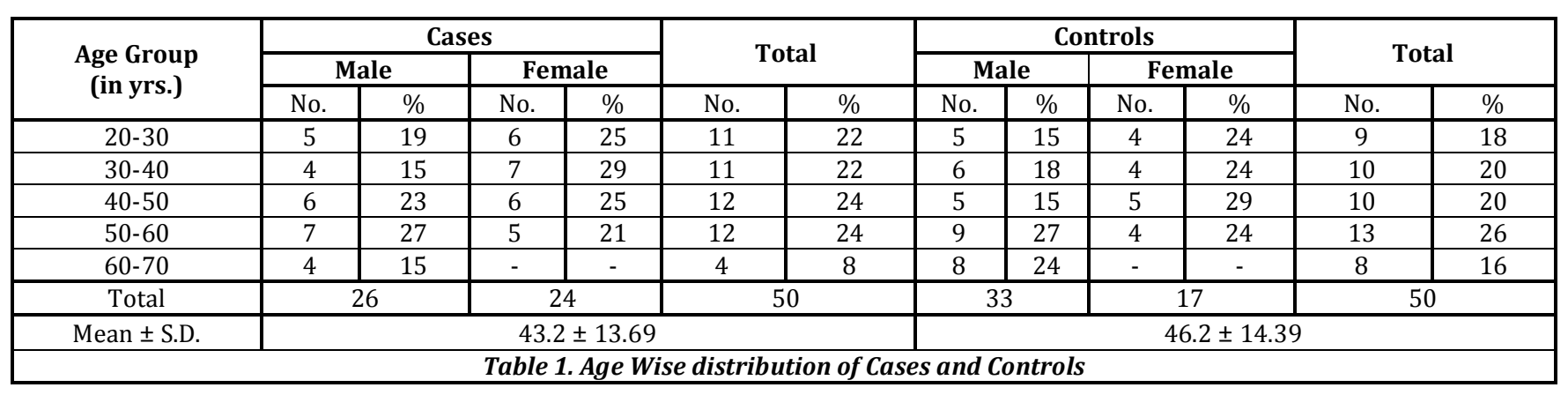

\begin{tabular}{|c|c|c|c|c|}
\hline \multirow{2}{*}{ Sex } & \multicolumn{2}{|c|}{ Cases } & \multicolumn{2}{c|}{ Control } \\
\cline { 2 - 5 } & No. & \% & 33 & No. \\
\hline Male & 26 & $42 \%$ & 17 & $66 \%$ \\
\hline Female & 24 & $48 \%$ & $54 \%$ \\
\hline Total & \multicolumn{2}{|c|}{ Table 2. Sex Wise distribution of Cases and Controls } \\
\hline \multicolumn{2}{|c|}{}
\end{tabular}

\begin{tabular}{|c|c|c|c|c|c|c|c|c|c|c|c|c|}
\hline \multirow{3}{*}{ Range (mg/dL) } & \multicolumn{4}{|c|}{ Cases } & \multirow{2}{*}{\multicolumn{2}{|c|}{ Total }} & \multicolumn{4}{|c|}{ Controls } & \multirow{2}{*}{\multicolumn{2}{|c|}{ Total }} \\
\hline & \multicolumn{2}{|c|}{ Male } & \multicolumn{2}{|c|}{ Female } & & & \multicolumn{2}{|c|}{ Male } & \multicolumn{2}{|c|}{ Female } & & \\
\hline & No. & $\%$ & No. & $\%$ & No. & $\%$ & No. & $\%$ & No. & $\%$ & No. & $\%$ \\
\hline $80-100$ & 4 & 15 & 2 & 8 & 6 & 12 & 5 & 15 & 4 & 24 & 9 & 18 \\
\hline $100-120$ & 6 & 23 & 4 & 17 & 10 & 20 & 6 & 18 & 3 & 18 & 9 & 18 \\
\hline $120-140$ & 4 & 15 & 2 & 8 & 6 & 12 & 5 & 15 & 5 & 29 & 10 & 20 \\
\hline $140-160$ & 6 & 23 & 5 & 21 & 11 & 22 & 8 & 24 & 3 & 18 & 11 & 22 \\
\hline $160-180$ & 4 & 15 & 6 & 25 & 10 & 20 & 5 & 15 & 2 & 12 & 7 & 14 \\
\hline $180-200$ & 2 & 8 & 0 & 0 & 2 & 4 & 1 & 3 & 0 & 0 & 1 & 2 \\
\hline $200-220$ & 0 & 0 & 4 & 0 & 4 & 8 & 1 & 3 & 0 & 0 & 1 & 2 \\
\hline 220 and above & 0 & 0 & 1 & 0 & 1 & 2 & 2 & 6 & 0 & 0 & 2 & 4 \\
\hline Total & \multicolumn{2}{|c|}{26} & \multicolumn{2}{|c|}{24} & \multicolumn{2}{|c|}{50} & \multicolumn{2}{|c|}{33} & \multicolumn{2}{|c|}{17} & \multicolumn{2}{|c|}{50} \\
\hline Mean \pm S.D. & \multicolumn{6}{|c|}{$147.22 \pm 38.71$} & \multicolumn{6}{|c|}{$138.32 \pm 49.54$} \\
\hline & \multicolumn{12}{|c|}{ Table 3. Serum Cholesterol Level in Cases and Controls } \\
\hline
\end{tabular}

${ }^{*} \mathrm{p}>0.05$ not significant 


\begin{tabular}{|c|c|c|c|c|c|c|c|c|c|c|c|c|}
\hline \multirow{3}{*}{ Range (mg/dL) } & \multicolumn{4}{|c|}{ Cases } & \multirow{2}{*}{\multicolumn{2}{|c|}{ Total }} & \multicolumn{4}{|c|}{ Control } & \multirow{2}{*}{\multicolumn{2}{|c|}{ Total }} \\
\hline & \multicolumn{2}{|c|}{ Male } & \multicolumn{2}{|c|}{ Female } & & & \multicolumn{2}{|c|}{ Male } & \multicolumn{2}{|c|}{ Female } & & \\
\hline & No. & $\%$ & No. & $\%$ & No. & $\%$ & No. & $\%$ & No. & $\%$ & No. & $\%$ \\
\hline $0-50$ & 1 & 4 & 1 & 4 & 2 & 4 & 1 & 3 & 0 & 0 & 1 & 2 \\
\hline $50-100$ & 11 & 42 & 9 & 38 & 20 & 40 & 19 & 58 & 9 & 53 & 28 & 56 \\
\hline $100-150$ & 8 & 31 & 7 & 29 & 15 & 30 & 7 & 21 & 8 & 47 & 15 & 30 \\
\hline $150-200$ & 4 & 15 & 5 & 21 & 9 & 18 & 5 & 15 & 0 & 0 & 5 & 10 \\
\hline $200-250$ & 0 & 0 & 1 & 4 & 1 & 2 & 0 & 0 & 0 & 0 & 0 & 0 \\
\hline $250-300$ & 2 & 8 & - & - & 2 & 4 & 1 & 3 & 0 & 0 & 1 & 2 \\
\hline 300 and above & 0 & - & 1 & - & 1 & 2 & 0 & 0 & 0 & 0 & 0 & 0 \\
\hline Total & \multicolumn{2}{|c|}{26} & \multicolumn{2}{|c|}{24} & \multicolumn{2}{|c|}{50} & \multicolumn{2}{|c|}{33} & \multicolumn{2}{|c|}{17} & \multicolumn{2}{|c|}{50} \\
\hline Mean \pm S.D. & \multicolumn{6}{|c|}{$126.34 \pm 64.83$} & \multicolumn{6}{|c|}{$105.66 \pm 38.55$} \\
\hline \multicolumn{13}{|c|}{ Table 4. Serum Triglyceride Level in Cases and Control Group } \\
\hline
\end{tabular}

${ }^{*} \mathrm{p}<0.05$. Comparison of cases and control group shows that $\mathrm{p}<0.05$ was moderately significant.

\begin{tabular}{|c|c|c|c|c|c|c|c|c|c|c|c|c|}
\hline \multirow{3}{*}{ Range (mg/dL) } & \multicolumn{4}{|c|}{ Cases } & \multirow{2}{*}{\multicolumn{2}{|c|}{ Total }} & \multicolumn{4}{|c|}{ Controls } & \multirow{2}{*}{\multicolumn{2}{|c|}{ Total }} \\
\hline & \multicolumn{2}{|c|}{ Male } & \multicolumn{2}{|c|}{ Female } & & & \multicolumn{2}{|c|}{ Male } & \multicolumn{2}{|c|}{ Female } & & \\
\hline & No. & $\%$ & No. & $\%$ & No. & $\%$ & No. & $\%$ & No. & $\%$ & No. & $\%$ \\
\hline $0-25$ & 2 & 8 & 0 & 0 & 2 & 4 & 0 & 0 & 1 & 6 & 1 & 2 \\
\hline $25-50$ & 5 & 19 & 1 & 4 & 6 & 12 & 7 & 21 & 3 & 18 & 10 & 20 \\
\hline $50-75$ & 8 & 31 & 7 & 29 & 15 & 30 & 8 & 24 & 5 & 29 & 13 & 26 \\
\hline $75-100$ & 9 & 35 & 11 & 46 & 20 & 40 & 12 & 36 & 8 & 47 & 20 & 40 \\
\hline $100-125$ & 2 & 8 & 3 & 13 & 5 & 10 & 4 & 12 & 0 & 0 & 4 & 8 \\
\hline $125-150$ & 0 & 0 & 1 & 4 & 1 & 2 & 1 & 3 & 0 & 0 & 1 & 2 \\
\hline $150-175$ & 0 & 0 & 0 & 0 & 0 & 0 & 0 & 0 & 0 & 0 & 0 & 0 \\
\hline $175-200$ & 0 & 0 & 1 & 4 & 1 & 2 & 0 & 0 & 0 & 0 & 0 & 0 \\
\hline 200 and above & 0 & 0 & 0 & 0 & 0 & 0 & 1 & 3 & 0 & 0 & 1 & 2 \\
\hline Total & \multicolumn{2}{|c|}{26} & \multicolumn{2}{|c|}{24} & \multicolumn{2}{|c|}{50} & \multicolumn{2}{|c|}{33} & \multicolumn{2}{|c|}{17} & \multicolumn{2}{|c|}{50} \\
\hline Mean \pm S.D. & \multicolumn{6}{|c|}{$74.74 \pm 28.84$} & \multicolumn{6}{|c|}{$76.40 \pm 37.00$} \\
\hline & & & Table & $D L L$ & I & sand & ntrols & & & & & \\
\hline
\end{tabular}

${ }^{*} p>0.05$ Not Significant.

\begin{tabular}{|c|c|c|c|c|c|c|c|c|c|c|}
\hline \multirow{3}{*}{ Range (mg/dL) } & \multicolumn{4}{|c|}{ Cases } & \multirow{2}{*}{\multicolumn{2}{|c|}{ Total }} & \multicolumn{4}{|c|}{ Controls } \\
\hline & \multicolumn{2}{|c|}{ Male } & \multicolumn{2}{|c|}{ Female } & & & \multicolumn{2}{|c|}{ Male } & \multicolumn{2}{|c|}{ Female } \\
\hline & No. & $\%$ & No. & $\%$ & No. & $\%$ & No. & $\%$ & No. & $\%$ \\
\hline $10-20$ & 13 & 50 & 11 & 0 & 24 & 48 & 20 & 61 & 10 & 59 \\
\hline $20-30$ & 8 & 31 & 7 & 29 & 15 & 30 & 9 & 27 & 6 & 35 \\
\hline $30-40$ & 3 & 12 & 4 & 17 & 7 & 14 & 3 & 9 & 1 & 6 \\
\hline $40-50$ & 0 & 0 & 1 & 4 & 1 & 2 & 0 & 0 & 0 & 0 \\
\hline $50-60$ & 2 & 8 & 0 & 0 & 2 & 4 & 1 & 3 & 0 & 0 \\
\hline 60 and above & 0 & 0 & 1 & 4 & 1 & 2 & 0 & 0 & 0 & 0 \\
\hline Total & \multicolumn{2}{|c|}{26} & \multicolumn{2}{|c|}{24} & \multicolumn{2}{|c|}{50} & & & \multicolumn{2}{|c|}{17} \\
\hline Mean \pm S.D. & \multicolumn{6}{|c|}{$25.50 \pm 12.56$} & \multicolumn{4}{|c|}{$21.26 \pm 7.96$} \\
\hline \multicolumn{11}{|c|}{ Table 6. VLDL Level in Cases and Controls } \\
\hline
\end{tabular}

$* \mathrm{p}<0.05$ Significant.

\section{DISCUSSION}

Gallstones have higher incidence in females ${ }^{8}$ due to the presence of endogenous and exogenous oestrogens (Given as oral contraceptive pills or hormone replacement therapy), which induce chenodeoxycholic acid and increased total bile acid pool with consequent saturation (Table 1). Also, progesterone causes smooth muscle relaxation and impairs emptying of gall bladder. ${ }^{9}$ Saadeldin $\mathrm{A}$ et $\mathrm{al}^{10}$ reported the high incidence of gallstones in the age group of $31-50$ years $(68.08 \%)$, while it was rare in patients $<30$ and elderly patients $>70$ years. A probable explanation for the higher prevalence of gallstones in these age groups was given by David $\mathrm{C}^{11}$ that with increasing age biliary cholesterol saturation increases due to decline in the activity of cholesterol 7á hydroxylase, the rate limiting enzyme for bile acid synthesis (Table 2). In the elderly, bile acid synthesis is reduced, biliary cholesterol output is increased and cholesterol saturation of bile increases.

Stones are usually classified into 3 groups depending upon their colours: pale yellow and whitish stones as cholesterol stones, black and blackish brown as pigment calculi and brownish yellow or greenish with laminated features as mixed calculi. There are studies which demonstrate the percentage of the types of gallstones present in patients. Ibtisam BM et al12 in their study found $53.85 \%$ as pigment stones, $29.23 \%$ mixed and $16.92 \%$ as cholesterol stones and these results were in agreement with Saadeldin A et $\mathrm{al}^{13}$ who considered that the most common type of gallstone in Sudanese patients was the pigment calculi 48 (51.07\%) followed by mixed stone (31.9\%) and then cholesterol type $(17 \%)$. Although, in our study we have not classified the stones according to the colours, but they have been classified according to their presence and absence in patients. 
In this study the levels of serum lipid profile; total cholesterol, triglycerides, high density lipoprotein cholesterol and low-density lipoprotein cholesterol were measured in patients and controls group. Comparison of serum lipid profile between patients and controls group showed that the levels of serum total cholesterol $(147.22 \pm 38.71)$ in patients were slightly higher than that of the control group (138.32 \pm 49.54$)$, but there was no significant variation in total cholesterol $(p>0.05)$ between patients and controls group (Table 3). These results were in agreement with Narjis et $\mathrm{al}^{8}$ and Naseem A et al ${ }^{14}$ who compared serum lipid profile between patients and controls group and showed that the levels of serum total cholesterol and LDL-C in patients were slightly higher than that of the control group, but there was no significant variation in total cholesterol and LDL-C ( $p>0.05)$ between patients and controls group. However, in our study LDL-C was even lower in patients $(74.74 \pm 28.84)$ as compared to control groups $(76.40 \pm 37.00)$ (Table 5). Similarly, HDL-C was slightly higher in patients $(47.78 \pm 15.47)$ than the control groups $(44.06 \pm 15.65)$, but it was also not significant. In our study, triglycerides $(126.34 \pm 64.83)$ and very low-density lipoprotein $(25.50 \pm 12.56)$ concentration had significant $(\mathrm{p}<0.05)$ increase in patients with gallstone formation compared with control (105.66 \pm 38.55 and $21.26 \pm 7.96$ respectively) (Table 4 and 6). These results were in confirmation with the earlier reports of Atman ${ }^{15}$ and Narjis HA et al in their studies between lipid profile and gallstone formation. Although, gallstones are formed from supersaturation of cholesterol in the bile, but high total cholesterol levels themselves are not necessarily associated with gallstones as suggested by Dennis $\mathrm{L}^{16}$ and Portincasa. ${ }^{17}$ Some evidence suggests that high levels of triglycerides may impair the emptying actions of the gallbladder. The mechanism by which cholesterol stones form is not fully understood, but are likely as the result of a complex alteration in hepatobiliary function. ${ }^{18}$

\section{CONCLUSION}

Gallstone diseases being common disorders, might have an association with abnormal lipids. This study is done to compare the serum lipid abnormalities in patients who have cholelithiasis with controls. The results showed moderate significance in regard to TGs and VLDL in formation of gallstones. Although, this small sample size study concluded that there is correlation between presence of gallstones and TGs as well as VLDL, but a larger sample size study is required to further strengthen the findings of this study. The understanding of various other risk factors is also essential to establish the formation of gallstones.

\section{REFERENCES}

[1] Aulakh R, Mohan H, Attri AK, et al. A comparative study of serum lipid profile and gallstone disease. Indian J Pathol Microbiol 2007;50(2):308-12.

[2] Nagaraj SK, Paul P, Kumar MK, et al. Risk factors and the biochemical evaluation of biliary calculi in rural Kolar, Karnataka, India: a rural perspective of an urban disease. Journal of Clinical and Diagnostic Research 2012;6(3):364-8.
[3] Kumari DJ, Krishna SH. Role of body mass index, physical activity and nutrients in cholelithiasis in Guntur, Andhra Pradesh. J Hum Ecol 2010;31(3):1515.

[4] Hasan BF, Muhi SA, Kh. Ibrahim NA. The association among serum leptin, insulin and insulin resistance in Iraqi patients with cholelithiasis. American Journal of Medicine and Medical Sciences 2015;5(1):20-5.

[5] Smith JL, Riottot M, Nathanson LK. Effect of statins on biliary lipids and cholesterol gallstones. Journal of Cardiology 2002;9(7-8):295-8.

[6] Tjandrajana E, Agarwal S, Danda S. Gallstones in a patient with homocystinuria. Indian J Gastroenterol 2009;28(4):157-8.

[7] Sanikidze T, Shengelia M, Chikvaidze E, et al. Mechanism of gallstones formation in women during menopause. Current Topics in Biophysics 2014;37:1-8.

[8] Narjis HAL, Sabah AAA. Biochemical and demographical study of lipid profile in sera of patients with gallstone. Iraqi Journal of Science 2012;53(2):760-8.

[9] Bennion LJ, Crundy SM. Risk factors for the development of cholelithiasis in man (second of two parts). The New England Journal of Medicine 1978;299(22):1221-7.

[10] Saadeldin AI, Kamal EE, Aamir AH, et al. Extensive quantitative analysis of gallstones. International Journal of Clinical Medicine 2014;5:42-50.

[11] David C. Textbook of surgery: the biological basis of modern surgical practice. $14^{\text {th }}$ edn. USA: WB Saunders Company 1991.

[12] Ibtisam BM, Idris OF, Modawe GA. Assessment of serum lipids profile in sudanese patients with cholelithiasis. Scholars Bulletin 2016;2(2):58-63.

[13] Saadeldin AI, Mohammed HFS, Kamal EE, et al. Prevalence of different types of gallstone in relation to age in Sudan. Sch J App Med Sci 2013;1(6):664-7.

[14] Naseem AC, Fatehuddin K, Allah BG, et al. Quantitative analysis of serum lipid profile in gallstone patients and controls. Pak J Anal Environ Chem 2010;11(1):59-65.

[15] Atman M. Study of lipid profile and bailiary composition in patient with gallstone. Msc. Thesis. Baghdad. 2006.

[16] Dennis LK, Stephen LH, Anthony SF, et al. Harrison's principles of internal medicine. $16^{\text {th }}$ edn. New York: McGraw-Hill Medical Publishing Division 2005.

[17] Portincasa P, Moschetta A, Palasciano G. Cholesterol gallstone disease. Lancet 2006;368(9531):230-9.

[18] Williams EJ, Green J, Beckingham I, et al. Guidelines on the management of common bile duct stones (CBDS). Gut 2008;57(7):1004-21. 